\title{
Análise de imunossupressores utilizados no contexto do transplante cardíaco: revisão de literatura
}

\author{
Analysis of immunosuppressors used in the context of heart transplantation: literature \\ review
}
Análisis de inmunosuppresores utilizados en el contexto del trasplante cardíaco: revisión de la literatura

Giovana Silva Correa Reis ${ }^{1 *}$, Estherfanny da Nóbrega Pinheiro ${ }^{1}$, Pedro Paulo Cardoso Assayag ${ }^{1}$, Lucas Oliveira Mota ${ }^{1}$, Andrícia de Jesus de Melo e Silva ${ }^{1}$, Arilson Lima da Silva ${ }^{1}$, Lucas Matheus da Silva Castro ${ }^{1}$, Henrique Custódio da Silva¹.

\begin{abstract}
RESUMO
Objetivo: Analisar descritivamente estudos que abordam os imunossupressores utilizados no contexto do transplante cardíaco. Métodos: Foram identificados 164 artigos, nas bases de dados Bireme, Pubmed, Scientic Electronic Library (Scielo), Literatura Latino-americana-Americana e do Caribe em Ciências da Saúde (LILACS) e Medical Literature Analysis and Retrievel System Online (MEDLINE), do quais foram incluídos 27, conforme critérios específicos. Resultados: Foram identificados fatores que influenciam na escolha terapêutica dos imunossupressores; dentre estes, destacam-se os efeitos adversos, os benefícios e as interações medicamentosas. Ademais, observou-se estudos que avaliam os benefícios de novos medicamentos. Considerações finais: Constatou-se a existência e a importância de diversos imunossupressores utilizados para aumentar a vida útil do enxerto cardíaco e reduzir as taxas de mortalidade. Contudo, alguns apresentam muitos efeitos adversos que limitam o uso destes. Assim, novos medicamentos ganham destaque, visto que buscam minimizar os tais efeitos, bem como aumentar a tolerância e a taxa de sobrevida dos pacientes submetidos ao procedimento.
\end{abstract}

Palavras-chave: Rejeição de enxerto, Tratamento farmacológico, Imunossupressores, Transplante de coração.

\section{ABSTRACT}

Objective: To analyze descriptively studies that address the immunosuppressants used in the context of heart transplantation. Methods: 164 articles were identified in databases Bireme, Pubmed, Scientic Electronic Library (Scielo), Literatura Latino-americana-Americana e do Caribe em Ciências da Saúde (LILACS) e Medical Literature Analysis and Retrievel System Online (MEDLINE), of which 27 were included, according to specific criteria. Results: Factors that influence the therapeutic choice of immunosuppressants have been identified. Among these, adverse effects, benefits and drug interactions stand out. In addition, studies have been found that assess the benefits of new drugs. Final considerations: the existence and importance of several immunosuppressants used to increase the life span of the cardiac graft and reduce mortality rates was verified. However, they have many adverse effects that limit their use. Thus, new drugs are highlighted, as they seek to minimize adverse effects as well as to increase patient tolerance and survival rates of patients undergoing the procedure.

Key words: Graft rejection, Drug therapy, Immunosuppressive agents, Heart transplantation.

\section{RESUMEN}

Objetivo: Analizar descriptivamente los estudios que abordan los inmunosupresores utilizados en el contexto del trasplante cardíaco. Métodos: Se identificaron 164 artículos en las bases de datos Bireme, Pubmed, Scientic Electronic Library (Scielo), Latin American-American and Caribbean Literature in Health Sciences (LILACS) y Medical Literature Analysis and Retrievel System Online (MEDLINE), de los cuales 27 fueron incluidos, según criterios específicos. Resultados: se identificaron los factores que influyen en la elección terapéutica de los inmunosupresores; entre estos, se destacan los efectos adversos, los beneficios y las interacciones farmacológicas. Además, se han realizado estudios que evalúan los beneficios de los nuevos fármacos. Consideraciones finales: Se encontró la existencia e importancia de varios inmunosupresores utilizados para aumentar la vida útil del injerto cardíaco y reducir las tasas de mortalidad. Sin embargo, algunos tienen muchos efectos adversos que limitan su uso. Así, se destacan los nuevos fármacos, que buscan minimizar dichos efectos, así como incrementar la tolerancia y la tasa de supervivencia de los pacientes sometidos al procedimiento.

Palabras clave: Rechazo de injerto, Quimioterapia, Inmunosupresores, Trasplante de corazón.

${ }^{1}$ Universidade do Estado do Pará (UEPA), Belém - PA. *E-mail: giovanareis1102@gmail.com 


\section{INTRODUÇÃO}

O transplante cardíaco é um procedimento de alta importância atualmente, sabe-se que são realizados cerca de 110 mil procedimentos ao redor de todo o mundo (BACAL F et al, 2018). Além disso, é válido ressaltar que este é indicado principalmente para pacientes com Insuficiência Cardíaca avançada os quais apresentem sintomas limitantes e que apesar de toda a terapêutica medicamentosa já aplicada, ainda possuam um prognóstico desfavorável. Destaca-se a necessidade de avaliar as relações de custo e efetividade para os receptores do órgão, considerando os riscos e os benefícios envolvidos na terapia, e de comparar a capacidade de benefício do paciente em comparação com outros possíveis destinatários, mais aptos à doação do órgão (BRAGHA SK, et al. 2018).

Ademais, apesar do sucesso que a terapia de transplante revela no período de sobrevida e na melhora da qualidade de vida, possíveis complicações também são observadas, dentre estas, tem-se como exemplos: a disfunção precoce do enxerto, vasculopatia do aloenxerto cardíaco, lesões renais aguda e crônica, infecção e, ainda, a possível rejeição do enxerto cardíaco. A rejeição do transplante é responsável por cerca de $5 \%$ da mortalidade nos primeiros 30 dias de pós-operatório e $10 \%$ entre o $31^{\circ}$ dia e 1 ano (POTENA L, et al., 2018). De maneira geral, existem padrões de rejeição de enxertos e sua classificação tem como base as características histopatológicas e o tempo decorrido durante o processo (ABBAS AK, et al., 2015).

Nesse sentido, em relação ao padrão histopatológico, pode ser agudo, hiperagudo e crônico. Sendo classificado como agudo quando os anticorpos pré-formados reativos com o endotélio vascular ativam o sistema complemento, desencadeando trombose intravascular rápida e necrose da parede do vaso; é hiperagudo quando os linfócitos T CD4+ e CD8+ reativos em associação com aloantígenos localizados nas células endoteliais e nas células do parênquima medeiam danos destes tipos celulares, aumentando a lesão parenquimatosa e vascular; e crônico, quando a lesão à parede do vaso conduz a proliferação celular do músculo liso e oclusão luminal. Esta lesão pode ser provocada por uma reação inflamatória crônica a aloantígenos na parede do vaso (ABBAS AK, et al., 2015).

No contexto do transplante cardíaco, a rejeição pode ocorrer de forma hiperaguda, aguda celular e aguda humoral. A rejeição hiperaguda é mediada por anticorpos pré-formados, geralmente dirigidos contra o sistema $\mathrm{ABO}$ ou o sistema antígeno leucocitário humano, sendo uma reação precoce, ocorrendo imediatamente ou apenas poucas horas após o TC (CRUZ CBBV, 2019). A rejeição aguda celular pode ocorrer a qualquer momento após o transplante e se caracteriza como uma resposta inflamatória células T mediada, a qual é a resposta mais frequente (MANGINI S, et al., 2015). A rejeição humoral pode ocorrer em duas situações: quando há a pré-sensibilização a antígenos anti-HLA ou não HLA ou quando acontece a produção de anticorpos de novo (BACAL F, et al., 2018).

Mesmo após a realização eficiente do transplante, o receptor possui diversos fatores de risco que podem ocasionar a complicação supracitada, de modo a reduzir o tempo de sobrevivência do enxerto. Para prevenir a rejeição aguda, é essencial iniciar a terapia de indução, a qual visa proporcionar imunossupressão de alta intensidade no período pós-operatório precoce (BRIASOULIS A, et al., 2018). Já a terapia de manutenção, objetiva aumentar a sobrevida dos pacientes transplantados, diminuindo episódios de rejeição e infecções oportunistas, ao auxiliar a terapêutica primária, nesse caso, a terapia de indução (BACAL F, et al., 2018). Com os avanços dessa terapia imunossupressora, os resultados dos transplantes cardíacos continuam a melhorar, apesar dos receptores cada vez mais velhos e apresentando um maior risco, sendo a sobrevida média do adulto após o procedimento equivalente a aproximadamente 10,7 anos (MCCARTNEY SL et al, 2017).

Assim, destaca-se especificamente o papel dos imunossupressores, os quais têm como objetivo prevenir a rejeição de enxertos além de minimizar possíveis efeitos adversos indesejáveis, tais como infecção, malignidade e toxicidade medicamentosa. O uso dessa classe de medicamentos é o que permite aos pacientes submetidos ao transplante um menor índice de rejeição aguda do transplante e um maior tempo de vida, garantindo a funcionalidade do enxerto. O regime de imunossupressores a serem utilizados depende do curso clínico individualizado do paciente, podendo este utilizar um, dois ou mais medicamentos ao longo da vida. Tem-se como exemplo de imunossupressores costumeiramente utilizados os agentes antiproliferativos, 
os inibidores da calcinerina, e os corticosteroides, muito presentes na terapia de manutenção (JASIAK EM e PARK JM, 2016; HOLT CD, 2017)

Assim, o presente artigo tem como objetivo analisar descritivamente estudos dos últimos 3 anos, que abordem os imunossupressores utilizados no contexto do transplante cardíaco.

\section{MÉTODOS}

O presente estudo foi desenvolvido a partir de uma revisão bibliográfica como suporte metodológico, a qual foi realizada por meio da seleção de artigos pertinentes acerca dos imunossupressores e seus usos no contexto do transplante cardíaco.

A busca eletrônica da presente revisão foi realizada nas bases de dados Pubmed, Scientic Electronic Library (Scielo) e Literatura Latino-americana-Americana e do Caribe em Ciências da Saúde (LILACS). Foram definidos como critérios de inclusão, documentos originais e de revisão, os quais abordavam as diversas terapias com imunossupressores nos transplantes de coração, publicados na íntegra e na forma online, no período de 2017-2020, nos idiomas português e inglês; foram utilizados como descritores: Graft rejection; Drug therapy; Immunosuppressive agents; Heart, respectivamente, em inglês e português. Estabeleceram-se como critérios de exclusão relatos de caso, pesquisas fora do tempo selecionado ou com ausência de enfoque no tema proposto.

A partir dessa busca eletrônica, foram encontrados 164 potencialmente relevantes e publicados em bases de dados nos últimos 3 anos, dos quais restaram 113, após eliminação dos artigos presentes, de modo concomitante em mais de uma base de dados. Por fim, após seleção por leitura dos resumos, segundo os critérios de inclusão e exclusão, o número final de artigos em texto completo avaliados para elegibilidade e também incluídos por síntese qualitativa foi 27 (Figura 1).

Figura 1 - Fluxograma dos resultados das buscas em bases de dados e os motivos de exclusão.



Fonte: Reis GSC, et al., 2021.

\section{RESULTADOS}

Após análise, 27 estudos encaixaram-se nos critérios de inclusão da pesquisa. A partir da leitura dos estudos foi possível a elaboração de um quadro que reúne as principais informações de cada artigo (Quadro 1). 
Quadro 1 - Distribuição dos artigos incluídos na revisão de acordo com o autor, ano de publicação e informações acerca do estudo.

\begin{tabular}{|c|c|c|}
\hline $\mathbf{N}$ & Autores (Ano) & Principais achados \\
\hline 1 & ASANTE-KORANG A, et al. (2017) & $\begin{array}{l}\text { Estudo retrospectivo. Buscou comparar os efeitos do Tacrolimus, Everolimus e Sirolimus. Evidenciou efeitos } \\
\text { nefrotóxicos e malignos. }\end{array}$ \\
\hline 2 & ASLEH R, et al. (2018) & $\begin{array}{l}\text { Estudo experimental. Testar a segurança e eficácia a longo prazo da conversão de inibidor de calcineurina em } \\
\text { sirolimus como terapia de manutenção na progressão e resultados da vasculopatia do aloenxerto cardíaco (VAC) } \\
\text { após transplante cardíaco. A conversão precoce ao sirolimus está associada à progressão atenuada da VAC e com } \\
\text { menor mortalidade a longo prazo e menos eventos relacionados ao VAC em comparação com o uso contínuo do } \\
\text { inibidor de calcineurina. }\end{array}$ \\
\hline 3 & AYASOUFI K, et al. (2018) & $\begin{array}{l}\text { Estudo experimental. Identificar se a inibição da aquaporina } 4 \text { melhora a sobrevivência de aloenxertos cardíacos } \\
\text { murinos totalmente incompatíveis com MHC submetidos a } 8 \text { horas de isquemia fria. O bloqueio da aquaporina } 4 \\
\text { reduziu significativamente a proliferação de células T e a produção de citocinas in vitro, sugerindo que a melhor } \\
\text { sobrevivência do enxerto é, pelo menos em parte, mediada através de efeitos diretos nas células T doadoras reativas. }\end{array}$ \\
\hline 4 & BUSZKO M, et al. (2017) & $\begin{array}{l}\text { Estudo laboratorial controlado. Buscou investigar o efeito do tratamento com baixa dose de Timoglobulina } \\
\text { exclusivamente em células T usando um modelo de camundongo transgênico CD3E humano BALB/c humano que } \\
\text { expressa receptores de células T humanas e murinas. Concluiu que a timoglobulina mostrou um efeito } \\
\text { imunossupressor. }\end{array}$ \\
\hline 5 & BUTTS RJ, et al. (2018) & $\begin{array}{l}\text { Estudo retrospectivo. Comparar duas estratégias de indução comuns, basiliximabe e ATG. Não } \\
\text { diferença na perda de enxerto na coorte basiliximab em comparação com a coorte ATG. A infecção antes da alta foi } \\
\text { mais comum na coorte ATG. A indução com ATG está associada à melhor sobrevida tardia do enxerto em } \\
\text { comparação com basiliximabe. }\end{array}$ \\
\hline 6 & DÍAZ-MOLINA B, et al. (2018) & $\begin{array}{l}\text { Estudo retrospectivo. Teve omo objetivo analisar a função imunológica dos pacientes com transplante cardíaco em } \\
\text { relação ao momento do início do everolimus e os resultados durante os primeiros meses após o transplante e a longo } \\
\text { prazo. Observo-se que apenas a conversão precoce do everolimus induz uma resposta imune pós-transplante. }\end{array}$ \\
\hline 7 & ERDOGAN I, et al. (2018) & $\begin{array}{l}\text { Estudo experimental. Descrever a experiência pessoal dos pesquisadores com o tratamento com rituximabe em } \\
\text { crianças com transplante cardíaco. Rituximabe pode ser usado na rejeição humoral após transplante de coração } \\
\text { pediátrico. No entanto, mais estudos são necessários para encontrar um tratamento eficaz para rejeição mediada } \\
\text { por anticorpos e rejeição celular resistente a esteroides em crianças. }\end{array}$ \\
\hline 8 & GONZÁLEZ-VÍLCHEZ F, et al. (2019) & $\begin{array}{l}\text { Estudo retrospectivo. Analisar episódios e infecções de rejeição aguda, os quais estão associados à sub e } \\
\text { superimunossupressão de tacrolimus, respectivamente. Tanto o uso de novo quanto o uso precoce de tacrolimus } \\
\text { parecem ter perfis de segurança e eficácia semelhantes aos da imunossupressão convencional baseada em } \\
\text { tacrolimus convencional. }\end{array}$ \\
\hline 9 & GONZÁLEZ-VÍLCHEZ F, et al. (2019) & $\begin{array}{l}\text { Estudo retrospectivo. Descrever a eficácia e segurança do uso do tacrolimus de liberação prolongada e precoce para } \\
\text { transplante cardíaco. A conversão de tacrollimus de liberação precoce para a de liberação prolongada em receptores } \\
\text { de transplante cardíaco na Espanha não foi associada a novas preocupaços de segurança e eficácia } \\
\text { imunossupressora apropriada. }\end{array}$ \\
\hline
\end{tabular}




\begin{tabular}{|c|c|c|}
\hline $\mathbf{N}$ & Autores (Ano) & Principais achados \\
\hline 10 & GUDE E, et al. (2017) & $\begin{array}{l}\text { Revisão sistemática. A introdução de novo de everolimus (Eve) em receptores de transplante cardíaco abre uma } \\
\text { redução precoce dos inibidores da calcineurina (CNI) e potencial de preservação da função renal, atenua a } \\
\text { progressão da vasculopatia do aloenxerto coronário e mantém a eficácia da rejeição. }\end{array}$ \\
\hline 11 & GUETA I, et al. (2019) & $\begin{array}{l}\text { Estudo retrospectivo. Investigar a influência da variabilidade do nível mínimo do Tacrolimus em resultados e rejeições } \\
\text { a longo prazo, durante e após o primeiro ano após o transplante cardíaco. Os pacientes no grupo de alta variabilidade } \\
\text { exibiram maior taxa de rejeição em longo prazo, sem diferença nos escores de rejeição no primeiro ano pós } \\
\text { transplante. A análise multivariada mostrou que a alta variabilidade do nível mínimo de tacrolimus foi associada a } \\
\text { um risco> } 8 \text { vezes maior de qualquer rejeição além do primeiro ano pós transplante. }\end{array}$ \\
\hline 12 & HEIM C, et al. (2018) & $\begin{array}{l}\text { Revisão sistemática. Elucidar esforços científicos visando diferentes novos mecanismos de aloenxerto cardíaco } \\
\text { vasculopatia e disfunção crônica do aloenxerto pulmonar. A prevenção da rejeição crônica melhoraria claramente a } \\
\text { sobrevivência dos pacientes após o transplante de coração e pulmão. }\end{array}$ \\
\hline 13 & JARMI T, et al. (2018) & $\begin{array}{l}\text { Estudo retrospectivo. Determinar o papel da globulina anti-timócito de coelho (rATG) na prevenção da rejeição e na } \\
\text { melhoria da sobrevida global. A indução com rATG não adiciona nenhum benefício de sobrevivência em } \\
\text { receptores de transplante de coração. Os pacientes que não receberam terapia de indução tinham maior expectativa } \\
\text { de vida em } 5 \text { e } 10 \text { anos. }\end{array}$ \\
\hline 14 & JENNINGS DL, et al. (2018) & $\begin{array}{l}\text { Revisão sistemática. Avaliar a eficácia e segurança dos inibidores de mTOR em pacientes com transplante cardíaco. } \\
\text { Pode-se atenuar o risco da vasculopatia de aloenxerto coronário e citomegalovírus em pacientes com transplante } \\
\text { cardíaco. Uma combinação mTOR/MMF preserva a função renal, mas aumenta o risco de rejeição celular aguda. }\end{array}$ \\
\hline 15 & KATZ M, et al. (2017) & $\begin{array}{l}\text { Estudo retrospectivo. Buscou explorar as tendências do risco de rejeição após transplante cardíaco nos últimos } 25 \\
\text { anos e sua relação com as mudanças no tratamento médico. Observou-se que as taxas de rejeição diminuíram nos } \\
\text { últimos } 20 \text { anos. }\end{array}$ \\
\hline 16 & KITTIPIBUL V, et al. (2017) & $\begin{array}{l}\text { Estudo experimental. Mostrar que a indução de doses baixas de basiliximabe no transplante cardíaco resulta em } \\
\text { resultados favoráveis de eficácia e segurança. Mostram que a indução de basiliximabe de baixa dose no transplante } \\
\text { cardíaco resultou em resultados favoráveis de eficácia e segurança. }\end{array}$ \\
\hline 17 & KHUSH KK, et al. (2017) & $\begin{array}{l}\text { Revisão sistemática. Apresentar abordagens para personalizar o atendimento de receptores de transplante cardíaco. } \\
\text { Existem estratégias que permitem aumentar os benefícios das terapias imunossupressoras e diminuir os seus ricos. }\end{array}$ \\
\hline 18 & LAUB MR, et al. (2018) & $\begin{array}{l}\text { Estudo retrospectivo. Avaliar a magnitude do impacto do clotrimazol nos requisitos de dosagem de tacrolimus para } \\
\text { manter os níveis objetivos. As doses de clotrimazol exercem uma interação significativa com tacrolimus que requer } \\
\text { monitoramento próximo e ajuste de dose. }\end{array}$ \\
\hline 19 & LIU J, et al. (2019) & $\begin{array}{l}\text { Estudo experimental. Procurou estabelecer uma estratégia eficiente de entrega de FK506 para o tratamento da } \\
\text { rejeição aguda após transplante cardíaco, combinando microbolhas carregadas com FK506 (FK506-MBs) com } \\
\text { destruição de microbolhas direcionadas por ultrassom. Concluiu-se que se trata de uma opção segura e livre de } \\
\text { radiação, com grande potencial. }\end{array}$ \\
\hline
\end{tabular}




\begin{tabular}{|c|c|c|}
\hline $\mathbf{N}$ & Autores (Ano) & Principais achados \\
\hline 20 & LÖFDAHL E e Rådegran G (2017) & $\begin{array}{l}\text { Revisão sistemática. Resumir o conhecimento atual sobre o comprometimento da força óssea após tranplante } \\
\text { cardíaco e terapia imunossupressora concomitante. Conclui-se que é necessário a elaboração de estratégias para } \\
\text { manejar e prevenir a osteoporose após o transplante cardíaco. }\end{array}$ \\
\hline 21 & LU C, et al. (2017) & $\begin{array}{l}\text { Estudo experimental. Buscou determinar se o tanshinol, um ingrediente do danshen, melhora a sobrevivência do } \\
\text { aloenxerto cardíaco e chegou-se a uma conclusão positiva. }\end{array}$ \\
\hline 22 & MILLER KK, et al. (2017) & $\begin{array}{l}\text { Estudo experimental. Teve como objetivo mostrar os efeitos do tratamento com talidomida em um modelo de } \\
\text { transplante aórtico crônico ortotópico em ratos. Não foram observados efeitos adversos, ademais, evidenciou-se que } \\
\text { o tratamento preveniu o desenvolvimento de Vasculopatia do Aloenxerto Coronário. }\end{array}$ \\
\hline 23 & MOCIORNITA AG, et al. (2018) & $\begin{array}{l}\text { Estudo experimental. Avaliar se as células do músculo liso vascular eram capazes de expressar o antígeno } \\
\text { leucocitário humano-G in vitro como resultado da exposição ao everolímus. Mostrou nova regulação do HLA-G pelo } \\
\text { everolimus, demonstrando a suprarregulação do HLA-G e a subsequente inibição da proliferação do HCASMC. Os } \\
\text { resultados apoiam a importância e o uso potencial do HLA-G no transplante cardíaco para terapia preventiva ou } \\
\text { como um marcador para identificar pacientes com alto risco de desenvolver da vasculopatia do enxerto. }\end{array}$ \\
\hline 24 & O’NEILL NA, et al. (2017) & $\begin{array}{l}\text { Estudo experimental. Procurou comparar a eficácia de } 3 \text { anticorpos monoclonais contemporâneos (mAb) que } \\
\text { bloqueiam a via de co-estimulação CD } 40 / C D 154 \text {, testados em um modelo de primata não humano pré-clínico. } \\
\text { Evidenciou-se que os anticorpos possuem diferentes níveis de eficácia em prolongar a vida do enxerto. }\end{array}$ \\
\hline 25 & SINGH AK, et al. (2018) & $\begin{array}{l}\text { Estudo experimental. Analisar células Treg (células D4 + CD25Hi FoxP3 }+\mathrm{T} \text { ) em linfócitos do sangue periférico de } \\
\text { receptores de babuínos a longo prazo que receberam xenoenxertos cardíacos geneticamente modificados de } \\
\text { doadores de suínos. As células T (Treg) regulatórias podem contribuir para prevenir ou retardar a rejeição do } \\
\text { xenoenxerto, controlando a ativação e expansão das células T reativas do doador, mascarando assim a resposta } \\
\text { imune antidoador, levando à sobrevivência a longo prazo dos xenoenxertos cardíacos. }\end{array}$ \\
\hline 26 & WEBB NJA, et al. (2019) & $\begin{array}{l}\text { Estudo experimental. Observou-se que o Ctrough é um marcador confiável para o monitoramento terapêutico de } \\
\text { medicamentos em receptores de transplante pediátrico tratados com grânulos de tacrolimus }\end{array}$ \\
\hline 27 & ZUCKERMANN A, et al. (2018) & $\begin{array}{l}\text { Revisão sistemática. Discutir dados recentes sobre o uso de inibidores da rapamicina em mamíferos (mTOR) no } \\
\text { transplante cardíaco. Efeitos benéficos dos mTORs contra o desenvolvimento da vasculopatia do enxerto, infecção } \\
\text { por citomegalovírus e malignidade foram mostrados. No entanto, a menor tolerabilidade da droga afetou o uso a } \\
\text { longo prazo em pacientes. }\end{array}$ \\
\hline
\end{tabular}

Fonte: Reis GSC, et al., 2021. 
A terapia de manutenção, em casos de transplante cardíaco, tem, como a maioria dos esquemas de imunossupressão utilizar três fármacos, sendo um corticosteroide (prednisona ou metilprednisona), um inibidor da calcineurina (ciclosporina ou tacrolimus) e um antiproliferativo (micofenolato ou azatioprina). O $33^{\circ}$ Registro da ISHLT mostra preferência pelo esquema tacrolimus/micofenolato e, nos pacientes com mais de 5 anos de seguimento, a tendência ao uso de combinações que incluam os inibidores da m-Tor (everolimus). Podem-se fazer necessários esquemas alternativos ou substitutivos, em três situações: falha no regime tríplice, toxicidade medicamentosa ou necessidade do uso de menor imunossupressão (BACAL F, et al., 2018).

Ademais, a taxa de sobrevivência de pacientes transplantados é limitada pelos efeitos adversos dos imunossupressores, tais como infecções e injúria renal. Por esse motivo, ainda é necessária a discussão de estratégias que maximizem o benefício destes fármacos (KHUSH KK, 2017).

\section{Comparações entre as terapias imunossupressoras}

Com base na toxicidade medicamentosa apresentada e na incidência de rejeição ao enxerto cardíaco, Katz M, et al. (2017) compararam as terapias mais recentes (Tacrolimus, Micofenolato e Everolimus) com as mais antigas (Azatioprina e Ciclosporina). Assim, obtiveram o resultado de que os pacientes submetidos a transplantes cardíacos com o uso das terapias mais recentes, possuem redução mais significativa no risco de rejeição ao enxerto, nas formas precoce (0-3 meses após o transplante), intermediária (3-12 meses após o transplante) e tardia (acima de 12 meses).

\section{Inibidores da Calcinerina: Tacrolimus}

A eficácia de uma cirurgia de transplante é possível devido ao uso de imunossupressores. Diante disso, destaca-se o Tacrolimus, o inibidor da calcinerina mais utilizado em todo o mundo, cada vez mais em ascensão. Webb NJA, et al. (2019) definiram que, para o monitoramento terapêutico em transplantes de coração pediátricos, por meio do $\mathrm{C}_{\text {trough, }}$ o qual pode ser utilizado como parâmetro de avaliação da exposição ao tacrolimus, visto que avalia o nível mínimo da concentração de um medicamento antes da administração da próxima dose. Ademais, há evidências que os inibidores da calcinerina, como tratamento em transplantes cardíacos pediátricos, devem ser descontinuados em pacientes com insuficiência renal, pois o uso do medicamento pode causar decréscimo na taxa de filtração glomerular.

González-Vílchez F, et al. (2019) encontrou menor nível de incidência de rejeição após conversão de um regime terapêutico IRT (immediate-release tacrolimus) para PRT (prolonged-release tacrolimus). No entanto, é válido ressaltar que esse benefício não é comprovado, dado que existe a possibilidade de os níveis de rejeição terem diminuído devido ao tempo decorrido após o transplante e não em virtude da mudança terapêutica em si.

Em relação a permuta entre as duas apresentações deste imunossupressor, seja a de liberação rápida ou prolongada, também foi demonstrado que não ocorre alteração do valor mínimo e dos níveis séricos mínimos (GONZÁLES-VÍLCHEZ F, et al., 2018). Esse é um fato importante a ser observado, uma vez que a variabilidade nos valores mínimos de tacrolimus indica um risco aumentado de rejeição a longo prazo (GUETA I, et al., 2018).

É inevitável que o Tacrolimus (TK506), quando administrado por via oral ou endovenosa, gere efeitos adversos, tais como nefrotoxicidade, neurotoxicidade e infecção. Por isso, são necessários mecanismos que evitem os efeitos da imunossupressão sistêmica. Assim, tem-se o uso de microbolhas alvo de ultrassom; estas consistem em gás de fluorocarboneto, encapsulado em uma concha de lipídeo ou de proteína, capazes de carrear medicamentos (LIU J, et al., 2019).

Ao entrarem em contato com as ondas de ultrassom, estas se rompem e liberam energia capaz de aumentar a permeabilidade tecidual, além de, concomitantemente, liberar o medicamento no tecido ao redor, aumentando a concentração da droga no órgão em questão e diminuindo a concentração nos outros órgãos, os quais são alvos ao utilizar vias de administração sistêmicas. Liu J, et al. (2019), mediante estudo experimental em ratos, descreveram o uso de TK506 por meio das microbolhas alvo de ultrassom, concluindo que estas diminuem os efeitos sistêmicos das citocinas, apesar de aumentar a concentração no tecido local. 
Durante o tratamento com imunossupressores após transplante, é essencial avaliar a possibilidade de iniciar uma profilaxia medicamentosa para prevenir eventuais infecções oportunistas. Contudo, deve-se prever interações medicamentosas, como a do clotrimazol (antifúngico utilizado para prevenção de candidíase oral) e o tacrolimus. Foi observado que a adição ou retirada do clotrimazol no regime terapêutico pode causar alterações nos valores mínimos do imunossupressor, podendo levar a um nível subterapêutico, o que seria prejudicial para o paciente (LAUB MR, et al., 2018).

Além do mais, o tacrolimus apresenta neurotoxicidade pouco frequente associada ao seu uso, a síndrome da encefalopatia reversível posterior, caracterizada por cefaleia, alteração do estado mental, convulsões e distúrbios visuais, identificando a dificuldade de manter a imunossupressão e gerenciar os efeitos adversos relacionados à medicação, levando em consideração o risco de rejeição aguda ao enxerto cardíaco (KAPOOR A, et al., 2017).

\section{Inibidores da m-Tor: Everolimus e Sirolimus}

A complicação de rejeição ao transplante cardíaco mais comum é a vasculopatia do aloenxerto coronário (VAC); por esse motivo, Gude E, et al. (2017) buscaram comprovar que a combinação de everolimus com exposição reduzida aos inibidores da calcineurina atenuam, consideravelmente, a VAC. Além disso, também foi demonstrado visível melhora na função renal, quando utilizado este novo esquema, juntamente com o tratamento das subsequentes 7-11 semanas após o transplante sem os inibidores da calcineurina, embora apresente o mesmo padrão de imunossupressão da terapia mais antiga..

Além disso, Mociornita AG, et al. (2018) constatou que o everolimus induz a expressão do antígeno leucocitário humano G (HLA-G) nas células musculares lisas. Ainda, segundo o estudo, tais células possuem importante papel no desenvolvimento da VAC. Ainda foi encontrado HLA-G solúvel no plasma de pacientes pós transplantados e os indivíduos observados não desenvolveram VAC em 15 anos após o transplante. A pesquisa indica que tanto 0 medicamento quanto 0 HLA-G possuem atividades antiproliferativas e imunossupressoras (LAUB MR, et al., 2018).

Outro medicamento da classe dos inibidores da m-Tor, o sirolimus, também está associado à progressão atenuada da VAC e seus eventos. Além disso, promove menor mortalidade a longo prazo, quando possui conversão precoce, ou seja, passa a ser utilizado antes de dois anos após o transplante, em detrimento ao uso contínuo de inibidores da calcineurina. Outros efeitos benéficos são: a ação eficaz contra o desenvolvimento de infecção por citomegalovírus e malignidade, e contra as reações também comuns quando há rejeição ao enxerto cardíaco (ASLEH R, et al., 2018).

No entanto, a menor tolerabilidade a esses medicamentos afetou o uso a longo prazo (ZUCKERMANN A, et al., 2018). Por esta razão, o tempo para iniciar o seu uso é algo a ser discutido; um estudo retrospectivo acerca do assunto concluiu que iniciar a sua utilização precocemente produz uma resposta antiviral adequada, apesar de não ser capaz de conter a resposta citotóxica, especialmente em relação ao everolimus (DÍAZMOLINA B, et al., 2018).

O sirolimus e o everolimus possuem menos efeitos colaterais, especialmente nefrotoxicidade e infecções, quando comparados a ciclosporina e ao tacrolimus; por esse motivo e com base em seus resultados, AsanteKorang A, et al. (2017) afirmam que a conversão para o uso dos inibidores da m-Tor, como imunossupressão primária, pode ser realizada com segurança. Ademais, essa classe medicamentosa, quando em combinação com Micofenolato de mofetila, representante da classe medicamentosa dos imunossupressores antiproliferativos, apresenta maior preservação da função renal, porém, aumenta o risco de rejeição celular aguda (JENNINGS DL, et al., 2018).

Löfdahl E e Rådegran G (2017) demonstraram que os agentes imunossupressores atuais, como os inibidores da m-Tor, também produzem complicações quanto ao seu uso, como força óssea comprometida e aumento do risco de fraturas ósseas são sinais comuns em pacientes que possuem osteoporose, no entanto, produzem bons resultados de sobrevida.

Outro estudo avaliou a prevalência de prejuízos cognitivos em pacientes transplantados, assumindo taxa de $40 \%$ na amostra estudada. Contudo, foi evidenciado que o uso de everolimo apresenta pouca ou nenhuma influência nas funções cognitivas dos pacientes (BÜRKER BS, et al., 2017). 


\section{Novos medicamentos em busca de mais efeitos benéficos}

A talidomida apresentou potencial de impedir o desenvolvimento da VAC, uma doença que afeta cerca de $30 \%$ dos pacientes transplantados cardíacos, 5 anos após o transplante, em experimentação realizada em ratos, sendo observada obliteração luminal reduzida, com resgate concomitante das células do músculo liso no meio aórtico dos enxertos (MILLER KK, et al., 2017). Ademais, O'Neill NA, et al. (2017) demonstraram como atenuar a VAC e prolongar a sobrevida do enxerto cardíaco, a partir do bloqueio do eixo CD154/CD40, parte essencial de reação imunológica na rejeição ao transplante, usando IDEC-131 (anticorpo monoclonal imunizado contra o CD154). Todavia, o estudo demonstrou tratamento imunomodulador inferior ao $5 \mathrm{C} 8 \mathrm{H} 1$ (anticorpo humano-rato contra o CD154) ou 2C10R4 (anticorpo humano-rato contra o CD40).

A globulina proveniente de coelhos (rabbit antithymocyte globulin) promove atraso na rejeição de 36 para 55 dias (JARMI T, et al., 2018). Buszko M, et al. (2017) também constatou o papel da globulina antitimócito (ATG) em atrasar o processo de rejeição, quando aplicada por via intravenosa, em ratos que haviam recebido transplantes cardíacos. Notou-se que os enxertos dos animais que receberam a globulina perduraram por 11 dias, em comparação ao grupo controle, no qual os enxertos sobreviveram por apenas 8 dias.

Tal fato concorda com o estudo de Butts RJ, et al. (2018) que, ao comparar a ATG com o Imunossupressor Basiliximab, demonstrou que a ATG é mais eficaz em prevenir o risco de rejeição antes da alta do paciente, ainda que, possua maior risco de perda do enxerto no período de um ano após o transplante.

O Tanshinol, principal componente do extrato de sálvia, também pode ser utilizado como imunossupressor sendo capaz de evitar a rejeição do enxerto transplantado e de estender a sua sobrevivência quando associado a rapamicina (LU C, et al., 2017).

Kittipibul V, et al. (2017) foram pioneiros ao evidenciar que a indução de doses baixas de basiliximabe no transplante cardíaco resultou em benefícios quanto à eficácia e segurança do medicamento, com baixos riscos de rejeição aguda do enxerto e poucos efeitos colaterais. Com isso, pode-se afirmar que o inicio do inibidor da calcineurina em população de baixo risco pode ser retardado com segurança, usando a estratégia de basiliximabe no pós-operatório em baixa dose modificada, demonstrando um movimento de novos estudos que tentam substituir as terapias antigas de imunossupressão, que tanto apresentam efeitos colaterais maléficos.

A busca por medicamentos mais eficazes contra as formas de rejeição após transplante cardíaco é contínua e progressiva. Erdogan I, et al. (2018) identificaram o uso comprovado do rituximabe na rejeição humoral, entretanto, foi possível observar que o sucesso do tratamento é controverso e se faz necessária a procura por um tratamento eficaz para a rejeição mediada por anticorpos e a rejeição celular resistente a esteroides.

Essa pesquisa impulsionou Ayasoufi K, et al. (2018) a obterem resultados que identificam as Aquaporinas (uma família de canais de água que facilitam a homeostase, lesão tecidual e inflamação) como um alvo promissor para diminuir a alorreatividade específica de doadores e melhorar a sobrevivência de transplante de órgãos de alto risco, como do coração, pois melhora a viabilidade das células do enxerto do doador, diminui as respostas das células $T$ reativas ao doador e estende a sobrevivência do aloenxerto na ausência de outra imunossupressão

Outros pesquisadores influenciados foram Singh AK, et al. (2018), os quais sugerem que o uso de Celulas $T$ reguladoras podem prevenir ou retardar a rejeição de xenoenxertos. A células $T$ reguladora é um pequeno subconjunto de células T CD4+ que possui função imunorreguladora e apresenta papel importante na supressão da reatividade imunológica.

O mecanismo protetor é demonstrado a partir do controle da ativação e expansão de células T reativas a doadores, mascarando a resposta imune e levando a sobrevivência a longo prazo de xenoenxertos cardíacos. Contudo, ainda é apresentado o principal obstáculo para a sobrevivência após o procedimento operatório: a rejeição crônica, uma doença imunomoduladora que, até o momento, não apresenta terapia eficaz (HEIM C, et al., 2018). 


\section{CONSIDERAÇÕES FINAIS}

Dessa forma, é possível ressaltar os principais fatores que influenciam na escolha terapêutica, como os efeitos adversos, os benefícios e as interações medicamentosas. Portanto, novos medicamentos ganham destaque, visto que buscam minimizar os efeitos adversos, bem como aumentar a tolerância e a taxa de sobrevida. Assim, melhoram a qualidade de vida do paciente. Por fim, vale ressaltar, ainda, que a produção de estudos a respeito dos métodos para prevenir a rejeição tardia do aloenxerto, são relevantes para a comunidade cientifica.

\section{REFERÊNCIAS}

1. ABBAS AK, et al. Imunologia celular e molecular. 8a ed. Rio de Janeiro: Elsevier; 2015.

2. ASANTE-KORANG A, et al. Conversion from calcineurin inhibitors to mTOR inhibitors as primary immunosuppressive drugs in pediatric heart transplantation. Clin Transplant, 2017; 31(10): e13054.

3. ASLEH R, et al. Long-Term Sirolimus for Primary Immunosuppression in Heart Transplant Recipients. J Am Coll Cardiol, 2018; 71(6): 636-50.

4. AYASOUFI K, et al. Aquaporin 4 blockade improves survival of murine heart allografts subjected to prolonged cold ischemia. Am J Transplant, 2018; 18(5): 1238-46.

5. BACAL F, et al. $3^{a}$ Diretriz Brasileira de Transplante Cardíaco. Arq Bras Cardiol. 2018;111(2):230-89.

6. BHAGRA SK, et al. Cardiac transplantation: indications, elegibility and current outcomes. Heart, 2019; 105 (3): 252 260.

7. BRIASOULIS A, et al. Induction immunosuppressive therapy in cardiac transplantation: a systematic review and metaanalysis. Heart Failure Reviews, 2018; 23(5): 641-9.

8. BÜRKER BS, et al. Cognitive function after heart transplantation: Comparing everolimus-based and calcineurin inhibitor-based regimens. Clin Transplant, 2017; 31(4): e12927.

9. BUSZKO M, et al. Differential depletion of total $T$ cells and regulatory $T$ cells and prolonged allotransplant survival in CD3E humanized mice treated with polyclonal anti human thymocyte globulin. PLOS ONE, 2017; 12(3): e0173088.

10. BUTTS RJ, et al. Comparison of basiliximab vs antithymocyte globulin for induction in pediatric heart transplant recipients: An analysis of the International Society for Heart and Lung Transplantation database. Pediatr Transplant, 2018; 22(4): e13190.

11. CRUZ CBBV. Avaliação de rejeição aguda em pacientes transplantados cardíacos pela técnica de speckle tracking (tese) - Universidade de São Paulo, São Paulo, 2019;

12. DíAZ-MOLINA B, et al. Early Everolimus Initiation Fails to Counteract the Cytotoxic Response Mediated by CD8+ T and NK Cells in Heart Transplant Patients. Front Immunol, 2018; 9: 2181.

13. ERDOGAN I, et al. Rituximab Therapy for Rejection in Pediatric Heart Transplant. Exp Clin Transplant, 2018; 16(2): 199-203.

14. GONZÁLEZ-VÍLCHEZ F, et al. Conversion From Immediate-Release Tacrolimus to Prolonged-Release Tacrolimus in Stable Heart Transplant Patients: A Retrospective Study. Transplantation Proceedings, 2019; 51: 1994-2001.

15. GONZÁLES-VÍLCHEZ $F$, et al. Eficacia y seguridad del uso de novo y precoz de tacrolimus de liberación prolongada en el trasplante cardiaco. Rev Esp Cardiol, 2018; 71(1): 18-25.

16. GUDE E, et al. Everolimus immunosuppression for renal protection, reduction of allograft vasculopathy and prevention of allograft rejection in de-novo heart transplant recipients: could we have it all?. Clin Transplant, 2017; 22(3): 198206.

17. GUETA I, et al. High tacrolimus trough level variability is associated with rejections after heart transplant. Am J Transplant, 2018; 18: 2571-8.

18. HOLT, CD. Overview of Immunosuppressive Therapy in Solid Organ Transplantation. Anesthesiology Clinics, 2017; 35 (3): $365-380$.

19. HEIM C, et al. New Targets for the Prevention of Chronic Rejection after Thoracic Organ Transplantation. Thorac Cardiovasc Surg, 2018; 66(1): 20-30.

20. JARMI T, et al. Outcomes of induction therapy with rabbit anti-thymocyt globulin inheart transplante recipients: a single center retrospective cohort study. Ann Transplant, 2018; 23: 422-6.

21. JASIAK NM, PARK JM. Immunosuppression in Solid-Organ Transplantation: Essentials and Practical Tips. Critical Care Nursing Quarterly, 2016; 39 (3):227-240.

22. JENNINGS DL, et al. Outcomes associated with mammalian target of rapamycin (mTOR) inhibitors in heart transplant recipients: A meta-analysis. Int J Cardiol, 2018; 265: 71-5.

23. KAPOOR A, et al. Posterior Reversible Encephalopathy Syndrome after Heart Transplantation: Diagnosis and Immunosuppressive Therapy. Tex Heart Inst J, 2017; 44(3): 205-8.

24. KATZ M, et al. Risk of early, intermediate, and late rejection following heart transplantation: Trends over the past 25 years and relation to changes in medical management. Tertiary center experience: The Sheba Heart Transplantation Registry. Clin Transplant, 2017; 31(10): e13063.

25. KHUSH KK. Personalized treatment in heart transplantation. Curr Opin Organ Transplant, 2017; 22(3): 215-20. 
26. KITTIPIBUL V, et al. Low-dose basiliximab induction therapy in heart transplantation. Clin transplant, 2017; 31(12): e13132.

27. LAUB MR, et al. Effects of Clotrimazole Troches on Tacrolimus Dosing in Heart Transplant Recipients. Transpl Infect Dis, 2018; 20(6): e12979.

28. LIU $\mathrm{J}$, et al. Improving acute cardiac transplantation rejection therapy using ultrasound-targeted FK506-loaded microbubbles in rats. Biomater Sci, 2019; 7(9): 3729-40.

29. LÖFDAHL E e RÅDEGRAN G. Osteoporosis following heart transplantation and immunosuppressive therapy. Transplant Rev (Orlando), 2017; 31(4): 232-9.

30. LU C, et al. Tanshinol suppresses cardiac allograft rejection in a murine model. J Heart Lung Transplant, 2017; 36(2): 227-36.

31. MANGINI S, et al. Transplante cardíaco: revisão. Einstein, 2015;13(2): 310-8.

32. MCCARTNEY SL, et al. Long-term outcomes and management of the heart transplant recipient. Best practice \& research. Clinical anaesthesiology, 2017; 31 (2): 237-248.

33. MILLER KK, et al. Thalidomide treatment prevents chronic graft rejection after aortic transplantation in rats - an experimental study. Transpl Int, 2017; 30(11): 1181-9.

34. MOCIORNITA AG, et al. Effects of everolimus and HLA-G on cellular proliferation and neutrophil adhesion in an in vitro model of cardiac allograft vasculopathy. Am J Transplant, 2018; 18: 3038-44.

35. O'NEILL NA, et al. Comparative Evaluation of aCD40 (2C10R4) and aCD154 (5C8H1 and IDEC-131) in a Nonhuman Primate Cardiac Allotransplant Model. Transplantation, 2017; 101(9): 2038-47.

36. POTENA L, et al. Complications of Cardiac Transplantation. Current Cardiology Reports, $2018 ; 20$ (9): 1-9.

37. SINGH AK, et al. CD4+CD25Hi FoxP3+ regulatory $\mathrm{T}$ cells in long-term cardiac xenotransplantation. Xenotransplantation, 2018; 25(2): e12379.

38. WEBB NJA, et al. Pharmacokinetics of tacrolimus granules in pediatric de novo liver, kidney, and heart transplantation: The OPTION study. Pediatr Transplant, 2019; 23(1): e13328.

39. ZUCKERMANN A, et al. mTOR Inhibition and Clinical Transplantation: Heart. Transplantation, 2018; $102(2 S)$ : S27-9. 